\title{
Joint Optimization of Transmission Policies for Collaborative Beamforming with Energy Harvesting Sensors
}

\author{
Lazar Berbakov, Carles Antón-Haro, and Javier Matamoros \\ Centre Tecnològic de Telecomunicacions de Catalunya (CTTC) \\ Parc Mediterrani de la Tecnologia, Av. Carl Friedrich Gauss 7, 08860 Castelldefels, Spain \\ E-mail: \{lazar.berbakov, carles.anton, javier.matamoros\}@cttc.es
}

\begin{abstract}
In this paper, we consider a scenario where a number of energy harvesting sensors cooperate to transmit a common message to a distant base station. The goal is to find the jointly optimal transmission (power allocation) policy which maximizes the total throughput for a given deadline. We solve the problem in a semi-analytical manner and rigorously prove its optimality. The computational complexity of the proposed scheme is analyzed in detail and compared with that of interior point methods. Performance is extensively assessed by means of computer simulations. As benchmarks, we consider the case where the transmission policies for each sensor are separately optimized, as well as other cluster-based suboptimal transmission strategies.
\end{abstract}

\section{INTRODUCTION}

$\mathbf{S}$ ENSOR nodes are typically powered by batteries that, quite often, are either costly, difficult or simply impossible to replace. Clearly, this limits network lifetime. Energy harvesting makes it possible to overcome this drawback by allowing sensors to harvest energy from e.g. solar, mechanical, or thermal sources. The harvested energy is typically stored in a device (e.g. battery, super capacitor) and then supplied for communication and/or processing tasks when needed. In recent years, many authors have analyzed how to optimally use such harvested energy. For single-sensor scenarios, in [1] the authors derive the optimal transmission (power allocation) policy which minimizes the time needed to deliver all data packets to the destination subject to causality constraints on energy and packet arrivals. In [2], the authors go one step beyond and, unlike [1], they consider finite storage capacity effects. In both cases, the energy harvesting instants and amounts of energy harvested are assumed to be non-causally known. Ozel et al generalize the analysis to Rayleigh-fading channels (instead of Gaussian) and for the case in which the information on the harvested energy and channel gains is either causally or non-causally known [3]. Other works in the literature have addressed scenarios with multiple energy harvesting terminals. This includes studies for the multiple-access [4], interference [5] and relay [6], [7] channels, as well as the broadcast channel with infinite [8] or finite battery capacity [9]. The impact of battery imperfections has also been addressed in a number of

This work is partly supported by the project JUNTOS (TEC2010-17816), NEWCOM\# (318306), Spanish Ministry of Education (FPU grant AP200803952) and by the Catalan Government under 2009 SGR 1046. works. For instance in [10] the authors compute the optimal transmission policy in the presence of (constant-rate) energy leakage in the battery. Going one step beyond, [11] generalizes the previous result to broadcast channels and, in addition, it identifies the associated online optimal transmission policy. A suboptimal policy exhibiting a notable performance is derived as well.

Collaborative beamforming techniques allow nodes in a Wireless Sensor Network (WSN) to act as a virtual antenna array in order to e.g. reach a distant Base Station (BS) or data sink. This, however, requires accurate frequency and phase synchronization over sensors. To that aim, one can resort to the iterative synchronization scheme with one-bit of feedback proposed in [12], or opportunistic sensor selection schemes [13].

\section{A. Contributions}

In this paper, we consider a scenario where a number of energy harvesting $(\mathrm{EH})$ sensors cooperate to transmit (beamform) a common message to a distant base station. This differs from the scenarios in [4], [5], where multiple messages were delivered to the destination node(s). The scenario is also different from that in our previous work [14] where the virtual array also comprised battery operated (BO) sensors. Specifically, our goal is to find the jointly optimal transmission (i.e., power allocation) policy which maximizes the total throughput for a given deadline. This problem is equivalent to that addressed e.g. in [1], [3] but here we consider the more general case with multiple transmitters. We also go one step beyond the distributed beamforming approaches in [12], [13] and MAC channel with common data [15] where all sensors were implicitly assumed to be battery operated, and investigate the impact of energy harvesting constraints on performance. Unlike [2], the storage capacity of the EH sensors is assumed to be infinite here and, differently from [10], the battery leakage effects negligible. The main contributions of this paper are as follows:

1) We pose the problem of computing the jointly optimal transmission policy in a convex optimization framework.

2) We derive a semi-analytical solution to the problem which leverages on (i) the computationally-efficient iterative coordinate descent method of [16]; and (ii) an algorithm that we propose in this paper, on which basis 
one can compute the optimal policy for one sensor whilst the policy(ies) for the remaining one(s) is (are) held fixed.

3) We rigorously prove the optimality of the proposed method for a virtual array with an arbitrary number of EH sensors. By doing so, we generalize the analysis in our previous work [14] where the virtual array comprised one EH sensor assisted by a BO one.

4) We analyze the computational complexity associated to the proposed optimization scheme. In this respect, interior point methods are used as a benchmark.

Besides, we extensively assess the performance of the proposed method in a realistic system scenario where vibrational energy is harvested from the environment.

\section{B. Organization}

The paper is organized as follows. In Section II, we introduce the signal and communication model for the two-sensor case. Next, in Section III we pose the optimization problem in a convex optimization framework. Then we show that it can be solved in an iterative fashion. In Section IV, we propose a semi-analytical algorithm which allows to compute the jointly optimal transmission policy for one particular sensor when that of the other one is held fixed. A rigorous proof of its optimality follows. Section V is devoted to generalize the previous results to networks with an arbitrary number of sensors. Then, in Section VI, we analyze and compare the computational complexity of the proposed optimization scheme. Next, we present selected computer simulation results in Section VII. Section VIII closes the paper by summarizing the main results and drawing some conclusions.

\section{Signal and Communication Model}

Let $I$ denote the total number of EH sensors which cooperate to transmit a common message to a distant base station. Initially, we restrict the analysis to the $t w o$-sensor case ${ }^{1}$ (i.e., $I=2$ ) and, thus, the received signal reads

$$
r(t)=m(t)\left(\sum_{i=1}^{2} w_{i}(t) e^{j \psi_{i}(t)}\right)+n(t)
$$

where the common message is given by $m(t)=\sum_{l} x_{l} g(t-$ $\left.l T_{s}\right)$, with $\left\{x_{l}\right\}$ standing for a sequence of zero-mean complex Gaussian symbols with unit variance ( $T_{s}$ is the symbol period) and $g(t)$ denoting the impulse response of a bandlimited pulse (unit bandwidth), that we have used for signal modulation (e.g., a sinc pulse); function $w_{i}(t)=\sqrt{p_{i}(t)} e^{j \phi_{i}(t)}$ denotes the $i$-th time-varying complex transmit weight to be designed (in polar notation); $e^{j \psi_{i}(t)}$ stands for the phase shift of the Gaussian sensor-to-base station channels ${ }^{2}$; and $n(t)$ is zeromean complex additive white Gaussian noise with unit variance (i.e. $n(t) \sim \mathcal{C N}(0,1))$. By properly designing $\phi_{i}(t)$ for

\footnotetext{
${ }^{1}$ This initial assumption, to be relaxed in Section V, greatly facilitates the presentation of the proposed algorithm.

${ }^{2}$ The effects of path-loss, slow and/or fast (block) fading could be specifically taken into account by modeling the received power as $p_{B F}(t)=$ $\left(\sum_{i=1}^{I} \alpha_{i}(t) \sqrt{p_{i}(t)}\right)^{2}$, with $\alpha_{i}(t)$ denoting the time-varying channel amplitude for the $i^{\text {th }}$ sensor-to-BS channel. As long as the $\alpha_{i}(t)$ coefficients were non-causally known, the optimal transmission policies could still be computed.
}

$i=\{1,2\}$, we assume that the aforementioned channel phase shifts and oscillator offsets can be ideally pre-compensated [12] (frequency and time synchronization are assumed, as well). With this assumption, the whole sensor network behaves as a virtual array capable of beamforming the message to the base station. The instantaneous received power at the base station is thus given by $p_{B F}(t)=\left(\sqrt{p_{1}(t)}+\sqrt{p_{2}(t)}\right)^{2}$, and the total throughput for a given deadline $T, G_{T}$, then reads

$$
G_{T}=G_{T}\left(p_{1}(t), p_{2}(t)\right)=\int_{0}^{T} \log \left(1+p_{B F}(t)\right) d t .
$$

Our goal is to find the jointly optimal transmission (power allocation) policies $p_{1}(t)$ and $p_{2}(t)$ such that $G_{T}$ is maximized subject to the causality constraints imposed by the energy harvesting processes, namely,

$$
\begin{aligned}
& e_{1}(t) \leq E_{1}(t) \triangleq \sum_{k: s_{k}<t} E_{1, k} \quad ; \quad 0 \leq t \leq T \\
& e_{2}(t) \leq E_{2}(t) \triangleq \sum_{k: s_{k}<t} E_{2, k} \quad ; \quad 0 \leq t \leq T,
\end{aligned}
$$

where $e_{1}(t)=\int_{0}^{t} p_{1}(\tau) d \tau$ and $e_{2}(t)=\int_{0}^{t} p_{2}(\tau) d \tau$ denote the energy consumption (EC) curves; and $E_{1}(t), E_{2}(t)$ stand for the cumulative energy harvesting $(\mathrm{cEH})$ constraints which, as Fig. 1 illustrates, are staircase functions. Besides, $E_{i, k}$ in the above expression accounts for the amount of energy harvested by sensor $i$ in the $k$-th event $(k=0 \ldots N-1)$. We define event $s_{k}$ as the time instant in which some energy is harvested by any of the sensors in the network $\left(E_{i, k}=0\right.$ for the sensor not harvesting any energy in that event). Both the events and the amounts of energy harvested $E_{i, k}$ are assumed to be non-causally known ${ }^{3}$. Further, we impose $E_{i, 0}>0$ for all $i$ (sensors) in such a way that collaborative transmission can start immediately.

Finally, we define epoch as the time elapsed between two consecutive events. Its duration is given by $\tau_{k} \triangleq s_{k}-s_{k-1}$ for $k=1 \ldots N-1$ and, likewise, we define $\tau_{N} \triangleq T-$ $s_{N-1}$. A given transmission policy is said to be feasible (yet, perhaps, not optimal) if, as imposed by (3) and (4), the energy consumption curves lie below the $\mathrm{cEH}$ ones at all times (or occasionally touch them).

\section{COMPUTATION OF THE OPTIMAL TRANSMISSION POLICY}

The following lemmas, the proofs of which can be found in our previous work [17], state the necessary conditions for a transmission policy to be optimal ${ }^{4}$.

Lemma 1. The transmit power in each sensor remains constant between consecutive events.

In other words, the transmit power in each sensor only potentially changes when new energy arrives to any of them.

Lemma 2. All the harvested/stored energy must be consumed by the given deadline $T$.

\footnotetext{
${ }^{3}$ Yet not realistic, the assumption of perfect knowledge makes this new problem (the interplay of energy harvesting and collaborative beamforming) more tractable. An online algorithm, where such knowledge is acquired over time, would require different approach to problem solving (e.g., stochastic programming).

${ }^{4}$ Some similar Lemmas can also be found in [1] or [4]
} 


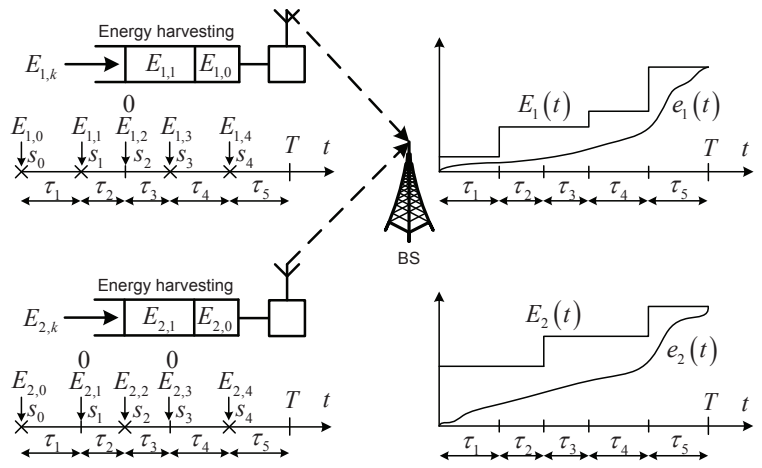

Fig. 1. Network with two energy harvesting sensors (left); cumulative energy constraints and energy consumption curves (right).

This means that, necessarily, the energy consumption curves touch the cumulative energy harvesting constraints at time instant $T$.

\section{Lemma 3. Transmit powers are strictly positive.}

That is, as soon as all sensors harvest some energy (namely, in $t=0$ as imposed earlier), there is no interruption in the data transmission of any sensor. That is, the total number of active sensors is constant and equal to $I$.

Lemma 1 above implies that $p_{1}(t)=p_{1, k}$ and $p_{2}(t)=p_{2, k}$ for $s_{k-1} \leq t<s_{k}$. That is, the power allocation curves $p_{1}(t)$ and $p_{2}(t)$ are necessarily staircase functions and, hence, the energy consumption curves $e_{1}(t)$ and $e_{2}(t)$ are piecewise linear. This observation allows to pose the original problem into a convex optimization framework in which a numerical or analytical solution are easier to find. Specifically, the original optimization problem given by the score function (2) and the causality constraints (3) and (4) can be re-written as

$$
\begin{aligned}
& \max _{\left\{p_{1, k}\right\}_{k=1}^{N},\left\{p_{2, k}\right\}_{k=1}^{N}} \sum_{k=1}^{N} \tau_{k} \log \left(1+\left(\sqrt{p_{1, k}}+\sqrt{p_{2, k}}\right)^{2}\right)(5) \\
& \text { s.t.: } \\
& \sum_{k=1}^{n} \tau_{k} p_{1, k} \leq E_{1}^{n}=\sum_{k=0}^{n-1} E_{1, k} \text { for } n=1 \ldots N \\
& \sum_{k=1}^{n} \tau_{k} p_{2, k} \leq E_{2}^{n}=\sum_{k=0}^{n-1} E_{2, k} \text { for } n=1 \ldots N \\
& p_{1, k}>0 \text { for } k=1 \ldots N \\
& p_{2, k}>0 \text { for } k=1 \ldots N
\end{aligned}
$$

where we have defined $E_{i}^{n} \triangleq E_{i}(t)$ for $t \in\left[s_{n-1}, s_{n}\right)$, and where the last two (strict) inequalities follow from Lemma 3 above. From Lemma 2, it becomes apparent that inequalities (6) and (7) must hold with equality for $n=N$. Besides, the problem is convex since (i) all the constraints are affine or linear; and (ii) the objective function can be shown to be concave. To prove the latter, we observe that the $k$-th term in the summation, namely $G_{k}\left(p_{1, k}, p_{2, k}\right)=\tau_{k} \log \left(1+\left(\sqrt{p_{1, k}}+\right.\right.$ $\left.\sqrt{p_{2, k}}\right)^{2}$ ), exclusively depends on the corresponding optimization variables $p_{1, k}$ and $p_{2, k}$ (i.e. no cross variables). Hence, it suffices to show that $G_{k}\left(p_{1, k}, p_{2, k}\right)$ is a concave function for any $k$. This can be easily verified by realizing that its $2 \times 2$ Hessian is negative definite, namely, $\nabla^{2} G_{k}\left(p_{1, k}, p_{2, k}\right) \prec 0$, for $p_{1, k}>0$ and $p_{2, k}>0$. From all the above, we conclude that the optimization problem (5)-(9) for the two-sensor case is strictly convex and, hence, its unique solution can be found at least numerically (e.g. by resorting to interior point methods). However, such numerical approaches can be computationally intensive, in particular when the number of energy harvesting events $N$ (i.e. the size of optimization vector) is large. For this reason, in the sequel we derive a semi-analytical solution which is computationally efficient. To that aim, we leverage on (i) the so-called iterative coordinate descent method (see next subsection); and (ii) the algorithm that we propose in Section IV, on which basis we can compute the optimal policy for one sensor whilst the policy of the other sensor is held fixed.

\section{A. Iterative Coordinate Descent Method}

Consider an optimization problem of the form:

$$
\begin{aligned}
& \max _{\mathbf{p}} f(\mathbf{p}) \\
& \text { s.t. } \mathbf{p} \in \mathcal{P},
\end{aligned}
$$

where $f(\mathbf{p}): \mathbb{R}^{n} \rightarrow \mathbb{R}$ is a continuously differentiable and concave function on a set $\mathcal{P}$ which, in turn, can be expressed as the Cartesian product of convex sets $\mathcal{P}_{1}, \ldots, \mathcal{P}_{n}$.

In [18], the authors prove that as long as (i) $\mathrm{f}(\mathbf{p})$ is a strictly concave function of the $i$-th coordinate of vector $\mathbf{p}$ (i.e. it has a unique maximum in $p_{i}$ when the remaining coordinates are held constant); and (ii) the sequence of said coordinates is generated according to an almost cyclic rule (or, more intuitively, every coordinate is iterated a sufficient number of times); an iterative procedure by which in each step a subset of coordinates in $\mathbf{p}$ are adjusted so as to minimize $f(\cdot)$ over $\mathcal{P}$ along this direction necessarily converges to the optimal solution.

One can easily prove that the optimization problem (5)(9) can be cast into the general framework described above $\mathrm{e}^{5}$ and, hence, it can be solved in an iterative fashion. In this context, we let $\mathbf{p}_{i}=\left[p_{i, 1}, p_{i, 2}, \ldots, p_{i, N}\right]^{T}$ denote a column vector gathering the $N$ components in the transmission policy $\left\{p_{i, k}\right\}_{k=1}^{N}$ of sensor $i \in\{1,2\}$. Further, we define transmission sub-policy as the subset of transmit powers $\left\{p_{i, k}\right\}_{k=k_{l}}^{k_{u}}$ in epochs $k=k_{l} \ldots k_{u}$. Accordingly, vector $\mathbf{p}_{i, k_{l}: k_{u}}=$ $\left[p_{i, k_{l}}, p_{i, k_{l}+1}, \ldots, p_{i, k_{u}}\right]^{T}$ gathers the transmit powers in such transmission sub-policy. Bearing all this in mind, the original optimization problem (5)-(9) can be decomposed in two individual sub-problems (one for each sensor in the network) and be iteratively solved. More precisely, in the $m$-th iteration we update the transmission policy of the first sensor, $\mathbf{p}_{1}^{(m)}$, by solving the subproblem

$$
\mathbf{p}_{1}^{(m)}=\underset{\mathbf{p}_{1}}{\arg \max } \sum_{k=1}^{N} \tau_{k} \log \left(1+\left(\sqrt{p_{1, k}^{(m)}}+\sqrt{p_{2, k}^{(m-1)}}\right)^{2}\right)
$$

s.t.:

$$
\begin{aligned}
& \sum_{k=1}^{n} \tau_{k} p_{1, k}^{(m)} \leq E_{1}^{n}=\sum_{k=0}^{n-1} E_{1, k} \text { for } n=1 \ldots N \\
& p_{1, k}^{(m)}>0 \text { for } k=1 \ldots N
\end{aligned}
$$

\footnotetext{
${ }^{5} \mathrm{~A}$ detailed derivation is omitted here due to space limitations.
} 
while holding fixed the policy for the second sensor that was computed in the previous iteration, namely, $\mathbf{p}_{2}^{(m-1)}$. Next, we update the transmission policy of the second sensor, $\mathbf{p}_{2}^{(m)}$ by solving

$$
\mathbf{p}_{2}^{(m)}=\underset{\mathbf{p}_{2}}{\arg \max } \sum_{k=1}^{N} \tau_{k} \log \left(1+\left(\sqrt{p_{1, k}^{(m)}}+\sqrt{p_{2, k}^{(m)}}\right)^{2}\right)
$$

s.t.:

$$
\begin{aligned}
& \sum_{k=1}^{n} \tau_{k} p_{2, k}^{(m)} \leq E_{2}^{n}=\sum_{k=0}^{n-1} E_{2, k} \text { for } n=1 \ldots N \\
& p_{2, k}^{(m)}>0 \text { for } k=1 \ldots N
\end{aligned}
$$

while holding $\mathbf{p}_{1}^{(m)}$ fixed $^{6}$. This procedure is iterated until a prescribed level of accuracy is attained or when the maximum number of iterations is reached. Since in each iteration both transmission policies are updated, the almost cyclic rule is clearly satisfied. To remark, this optimization technique has been widely used in multi-user scenarios (see e.g. [4] and [19] for details).

\section{OPTIMIZATION OF THE TRANSMISSION POLICY OF ONE EH SENSOR}

In this section, we propose an algorithm to semi-analytically find the (jointly) optimal transmission policy of one sensor whilst that of the other one is held fixed.

\section{A. Proposed algorithm}

Assume, without loss of generality, that we want to optimize the transmission policy of the first sensor. The Karush-KuhnTucker (K.K.T.) conditions for the sub-problem (11)-(13), $\operatorname{read}^{7}$ :

$$
\begin{aligned}
\frac{\partial \mathcal{L}_{1}}{\partial \breve{p}_{1, k}} & =0 \\
\sum_{k=1}^{n} \tau_{k} \breve{p}_{1, k} \leq E_{1}^{n} & =\sum_{k=0}^{n-1} E_{1, k} \text { for } n=1 \ldots N \\
\breve{p}_{1, k} & >0 \\
\breve{\lambda}_{n}, \breve{\mu}_{k} & \geq 0 \\
\breve{\lambda}_{n}\left(\sum_{k=1}^{n} \tau_{k} \breve{p}_{1, k}-E_{1}^{n}\right) & =0 \text { for } n=1 \ldots N \\
-\breve{\mu}_{k} \breve{p}_{1, k} & =0,
\end{aligned}
$$

where $\frac{\partial \mathcal{L}_{1}}{\partial \breve{p}_{1, k}}$ stands for the partial derivative of the Lagrangian associated to the sub-problem, namely,

$$
\begin{aligned}
\frac{\partial \mathcal{L}_{1}}{\partial \breve{p}_{1, k}}= & -\tau_{k} \frac{\sqrt{\breve{p}_{1, k}}+\sqrt{p_{2, k}}}{\sqrt{\breve{p}_{1, k}}\left(1+\left(\sqrt{\breve{p}_{1, k}}+\sqrt{p_{2, k}}\right)^{2}\right)} \\
& +\tau_{k}\left(\sum_{n=k}^{N} \breve{\lambda}_{n}-\frac{\breve{\mu}_{k}}{\tau_{k}}\right) .
\end{aligned}
$$

\footnotetext{
${ }^{6}$ Note that each subproblem includes only its own energy causality constraint. This follows from the fact that (i) causality for the other (fixed) transmission policy is enforced in the previous iteration; and (ii) causality constraints are not mutually coupled since both sensors harvest energy independently.

${ }^{7}$ For brevity, hereinafter we omit the iteration index $m$.
}

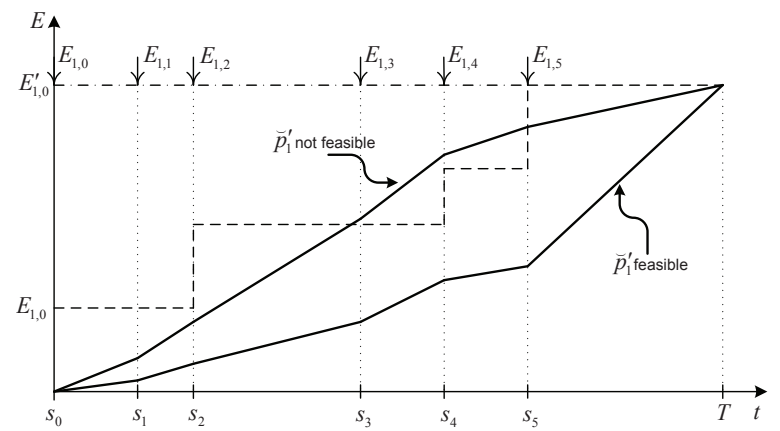

Fig. 2. Original and virtual optimization problems. Dashed and dash-dotted lines account for the original and virtual cEH curves, respectively.

The complementary slackness condition (22) along with (19) imply that, at the optimal point $\breve{\mathbf{p}}_{1}$, all $\breve{\mu}_{k}$ must vanish, i.e. $\breve{\mu}_{k}=0, \forall k$. Hence, by defining

$$
A_{k}=\sum_{n=k}^{N} \lambda_{n}
$$

the partial derivative of the Lagrangian in (23) can be conveniently re-written as

$$
\frac{\partial \mathcal{L}_{1}}{\partial \breve{p}_{1, k}}=-\tau_{k} \frac{\sqrt{\breve{p}_{1, k}}+\sqrt{p_{2, k}}}{\sqrt{\breve{p}_{1, k}}\left(1+\left(\sqrt{\breve{p}_{1, k}}+\sqrt{p_{2, k}}\right)^{2}\right)}+\tau_{k} \breve{A}_{k} .
$$

In order to compute the optimal transmission policy for the first sensor, we partly leverage on (and generalize) the algorithm presented in our previous work [14]. The algorithm allows to analytically compute the optimal policy for a battery operated (BO) sensor in a virtual array composed of one $\mathrm{EH}$ plus one BO sensor (see Appendix A). For a BO sensor, we have $E_{i, 0}>0$ whereas $E_{i, k}=0$ for $k=1 \ldots N-1$, In other words, no energy other than that initially stored in its battery is harvested during sensor operation. As a result, the cEH curve defined in (3) or (4) is no longer a staircase function. Instead, it takes a constant value for the whole $0 \leq t \leq T$ period.

Specifically, in order to compute the optimal transmission policy for the first sensor we propose the following 4-step procedure:

1) Check whether a BO-like solution for epochs $k=$ $1 \ldots N$ is feasible: To that aim, we assume that all the energy harvested by the first sensor during its operation is available from $t=0$ (see Fig. 2). This is equivalent to solve an optimization sub-problem where we replace the set of actual harvested energies $E_{1, k}$ by the following virtual ones

$$
\begin{aligned}
& E_{1,0}^{\prime}=\sum_{k=0}^{N-1} E_{1, k} \\
& E_{1, k}^{\prime}=0
\end{aligned}
$$

for $k=1 \ldots N-1$. The corresponding virtual transmission policy $\breve{\mathbf{p}}_{1}^{\prime}$ can thus be computed as it is explained in the Appendix A. If such virtual transmission policy verifies the constraints (12)-(13) of the original sub-problem (i.e. it is feasible) then it is solved and the procedure 
terminated ${ }^{8}$. This follows from the facts that (i) the score function optimized in the virtual and original subproblems are identical; (ii) the sub-problem is strictly convex and, hence, the optimal solution is unique; and (iii) the solution of the virtual sub-problem is feasible for the original one too ${ }^{9}$. In other words, we have $\breve{\mathbf{p}}_{1}=\breve{\mathbf{p}}_{1}^{\prime}$. Interestingly enough, if the BO-like solution is feasible (and, thus, optimal) then $\breve{\mathbf{p}}_{1}$ is not affected by the causality constraints for $n=1 \ldots N-1$ which could well be dismissed. More formally, this is equivalent to say that, in the Lagrangian dual we have $\vec{\lambda}_{n}=0$ for $n=1 \ldots N-1$ (still, we must have $\breve{\lambda}_{N}>0$ in order to fulfill the necessary optimality condition given by Lemma 2 and, thus, enforce $\sum_{k=1}^{N} \tau_{k} \breve{p}_{1, k}=E_{1}^{N}$ in the KKT condition given by (21)).

On the contrary, if the resulting virtual policy $\breve{\mathbf{p}}_{1}^{\prime}$ violates one or more causality constraint given by (12) then it is not feasible. In order to enforce those constraints, we necessarily have $\breve{\lambda}_{n}>0$ for one or more epochs in $n=1 \ldots N-1$ (in addition to having $\breve{\lambda}_{N}>0$ ). Consequently, the optimal policy $\breve{\mathbf{p}}_{1}$ is such that the EC curve touches the $\mathrm{cEH}$ constraints in one or more corner points of the cEH curve in $n=1 \ldots N-1$. This case is addressed in Step 2.

2) Find the largest $L<N$ such that a BO-like solution for epochs $k=1 \ldots L$ is feasible and, simultaneously, the energy harvested by that sensor in $s_{L}$ is non-zero: If the BO-like virtual sub-policy $\mathbf{p}_{1,1: L}^{\prime}$ is feasible for original problem, then it verifies the causality constraints for $k=1 \ldots L$ (or, equivalently, the KKT conditions for such epochs). Hence, $\mathbf{p}_{1,1: L}^{\prime}$ is a good candidate for the optimal transmission policy $\breve{\mathbf{p}}_{1}$ since, at least, it verifies the problem constraints up to the $L$-th epoch. As soon as this happens, we move to Step 3 below (otherwise, we try a smaller value of $L$ ). It is worth noting that, analogously to Step 1, the fact that this virtual sub-policy is feasible implies that, in the Lagrangian dual, we have $\lambda_{n}=0$ for $n=1 \ldots L-1$ and $^{10} \lambda_{L}>0$. In other words, in the first $L$ epochs the EC curve associated to this candidate hits the cEH curve in $s_{L}$ only.

The reason why we impose the sensor to effectively harvest some energy in $s_{L}$ (i.e., $E_{1, L}>0$ ) will be clarified in Step 4 below. For the time being, it suffices to say that imposing $E_{1, L}>0$ is equivalent impose that the cEH curve has a corner point in $s_{L}$.

3) Retain sub-policy $\mathbf{p}_{1,1: L}^{\prime}$ and repeat Steps 1 and 2 for epochs $k=L+1 \ldots N$ only (instead of $k=1 \ldots N$ ). Steps 1 to 3 are iterated until all the transmit powers for epochs $k=1 \ldots N$ have been computed as BO-like feasible solutions.

4) Construct the optimal transmission policy as a con-

\footnotetext{
${ }^{8}$ Note that the generalized water-filling solution of [4] would only come to this conclusion after computing all waterlevels and realizing that they are equivalent

${ }^{9}$ Intuitively, since in a BO-like policy all the energy harvested is available from the onset the throughput it attains is necessarily higher than that of any other policy computed with the actual energy arrivals.

${ }^{10}$ This is consistent with the fact that, from Step 1, we know that there exists at least one $n<N$ such that $\breve{\lambda}_{n}>0$.
}

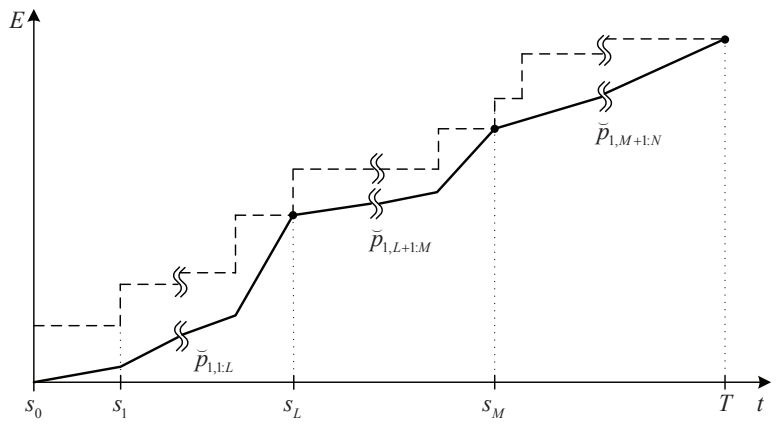

Fig. 3. Concatenation of sub-policies.

catenation of the BO-like sub-policies computed and retained in Steps 1-3. This extent is illustrated in Fig. 3 where, as an example, we have $\breve{\mathbf{p}}_{1}=$ $\left[\mathbf{p}_{1,1: L}^{\prime T}, \mathbf{p}_{1, L+1: M}^{\prime T}, \mathbf{p}_{1, M+1: N}^{\prime T}\right]^{T}$ with $L, M<N$. The intuition behind this approach is that, as previously discussed, each BO-like policy attains the highest possible throughput in its own epoch subset, which is determined in Steps 1-2. However, its overall optimality needs to be proved.

As a final remark, note that should the sub-policy $\mathbf{p}_{1,1: L}^{\prime}$ hit the cEH curve in a non-corner point, we would unavoidably have $p_{1, L+1}^{\prime}=0$ which contradicts the necessary optimality condition of Lemma 3 . This is why in Step 2 above we imposed $E_{1, L}>0$.

\section{B. Algorithm Optimality}

In the remainder of this section, we prove our claim that the resulting transmission policy is indeed optimal. This motivates the following two lemmas and theorem.

Lemma 4. If $L \leq N$ is the largest value for which a candidate BO-like transmission sub-policy $\mathbf{p}_{1,1: L}$ ending in a corner point of the cEH curve is feasible, then there exists no other transmission sub-policy $\mathbf{p}_{1,1: R}$ with $R<L$ such that it is part of the optimal transmission policy.

This lemma implies that, as soon as the aforementioned largest $L$ has been identified, there is no need to search for additional candidate sub-policies in which the EC curve touches the $\mathrm{cEH}$ one in a corner point at a previous time instant $s_{R}$ (since, for sure, the overall optimal transmission policy will not include those corner points). This avoids conducting an exhaustive search over events and, hence, allows us to move from Step 2 to Step 3 as described in the procedure above without compromising optimality. This lemma can be easily proved by contradiction, as shown in the Appendix B.

To insist, this Lemma does not state that $\mathbf{p}_{1,1: L}$ is part of the overall optimal policy (to that aim we need Lemma 5 next) but, instead, that $\dot{\mathbf{p}}_{1,1: L}$ cannot be part of it.

Lemma 5. If $L \leq N$ is the largest value for which a candidate BO-like sub-policy $\mathbf{p}_{1,1: L}$ ending in a corner point of the $c E H$ curve is feasible then such sub-policy is necessarily part of the optimal transmission policy $\breve{\mathbf{p}}_{1}$.

Proof: For the $L=N$ case, the proof is trivial. In order to prove the lemma for $L<N$, notice that from Step 1 we 
know that there exists one (or more) $\breve{\lambda}_{k}>0$ for $k=1 \ldots N-$ 1. In other words, the EC curve hits the cEH curve in some corner point(s) at $s_{k}$ with $k=1 \ldots N-1$. Assume that this occurs for the first time at $k=N-1$. If so, the corresponding BO-like sub-policy is feasible and $\lambda_{N-1}>0$. Otherwise, we know that the EC curve hits the cEH one for the first time in some corner point(s) at $s_{k}$ for $k=1 \ldots N-2$ for which $\lambda_{k}>0$. We recursively apply this procedure and, for $k=$ $L+1$, we realize that the corresponding BO-like policy is again not feasible and, thus, the first $\lambda_{k}>0$ is necessarily in the range $k=1 \ldots L$. Finally, for $k=L$ the BO-like policy is feasible and, from Lemma 4, we know that the EC curve associated to the optimal transmission policy does not touch the cEH one in a corner point at a previous time instant. Therefore only the BO-like transmission sub-policy $\mathbf{p}_{1,1: L}$ is in a position to satisfy the $\lambda_{k}>0$ for some $k=1 \ldots L$ and, thus, it must be part of the overall optimal transmission policy ${ }^{11}$. This concludes the proof.

Theorem 1. When the transmission policy for the second sensor is held fixed, the jointly optimal transmission policy for the first sensor, $\breve{\mathbf{p}}_{1}$, can be computed with the procedure given in Algorithm 1.

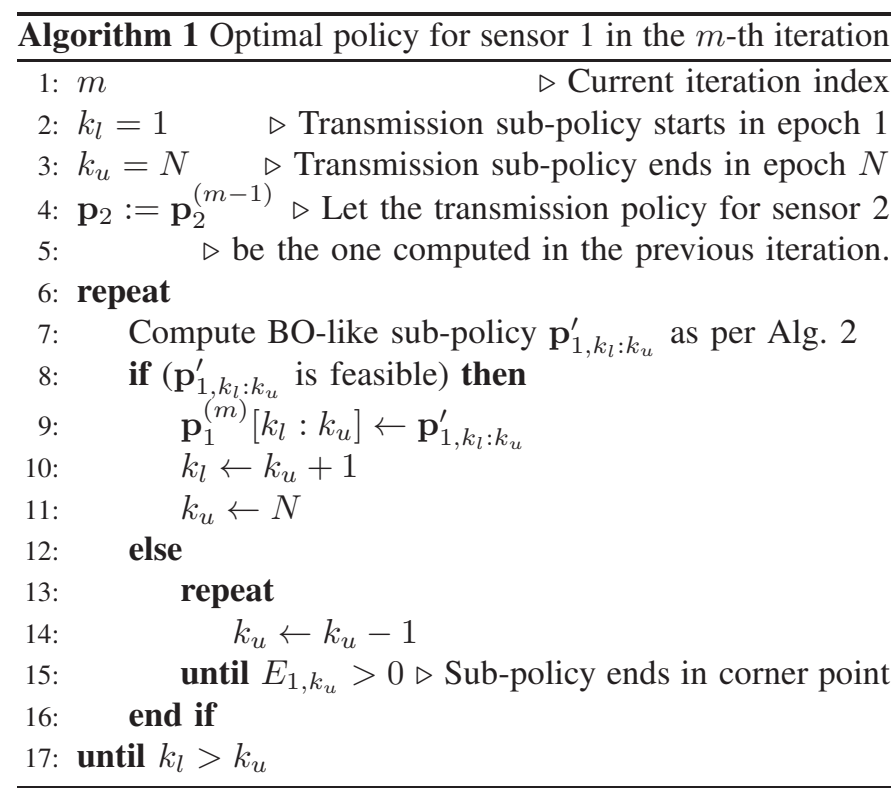

Algorithm 1 is nothing but a more formal representation of the 4-step procedure described above. The corresponding proof of its optimality follows.

Proof: After computing and retaining the candidate sub-policy $\mathbf{p}_{1,1: L}$, we only need to compute the remaining elements of the optimal transmission policy, namely, $\breve{p}_{1, k}$ for $k=L+1 \ldots N$. Since at $s_{L}$ the total energy spent equals the energy harvested, those elements exclusively depend on the energy harvested in subsequent events (i.e. not in the previous ones, or on the optimal transmit powers for the preceding epochs). Thus, we can simply re-start the 4-step procedure above for epoch $L+1$ onwards, as it is done in Step 3. The

\footnotetext{
${ }^{11}$ Note that, unlike [4], we have proven that the overall optimal policy can be computed as a concatenation of the "longest" feasible BO-like sub-policies.
}
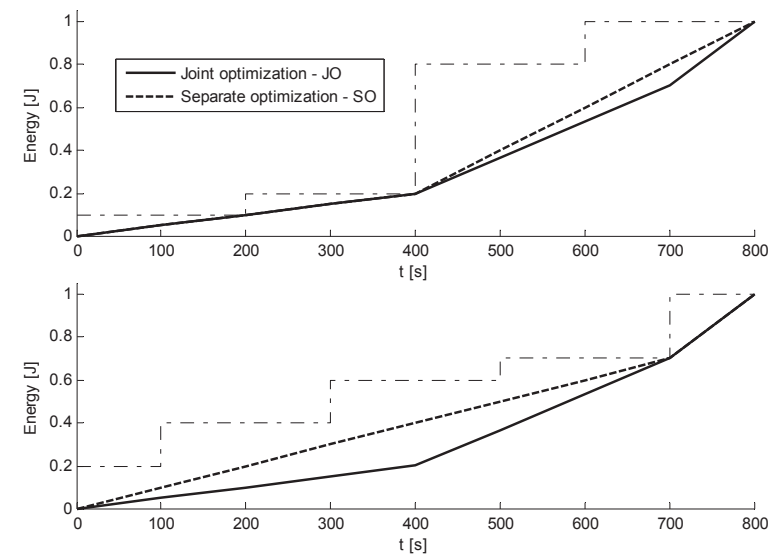

Fig. 4. Joint vs. separate optimization of transmission policies $(I=2)$

overall optimal transmission policy is finally computed as a concatenation of the BO-like sub-policies computed in Steps $1-3$, which is accomplished in Step 4.

To conclude this section, in Figure 4 we depict the transmission policies of both sensors when (i) both policies are jointly optimized (JO); and (ii) such policies are separately optimized (SO) as in the single-sensor scenario addressed in [1], which is suboptimal. For case (ii), each sensor attempts to maximize its own throughput rather than the throughput resulting from cooperative transmission. In these circumstances, the "optimal" transmission policy for each sensor can be conveniently computed as the shortest string lying below the cEH curve of that sensor (see [1], for details). The impact in terms of throughput will be assessed in detail in Section VII.

\section{Generalization to Networks with an Arbitrary NUMBER OF SENSORS}

For the general case of a network with $I$ energy-harvesting sensors, the optimization problem reads

$$
\begin{aligned}
\max _{\mathbf{p}_{1}, \mathbf{p}_{2}, \ldots \mathbf{p}_{I}} \sum_{k=1}^{N} \tau_{k} \log \left(1+\left(\sum_{i=1}^{I} \sqrt{p_{i, k}}\right)^{2}\right) \\
\text { s.t.: } \\
\sum_{k=1}^{n} \tau_{k} p_{i, k} \leq E_{i}^{n}=\sum_{k=0}^{n-1} E_{i, k} \quad i=1 \ldots I \\
p_{i, k}>0 \quad \begin{aligned}
n & =1 \ldots N \\
& =1 \ldots I \\
k & =1 \ldots N .
\end{aligned}
\end{aligned}
$$

In order to generalize the results from the previous sections, it suffices to (i) show that the score function (28) is strictly concave as well; and (ii) ensure that the sequence of transmission policy updates of the iterative coordinate descent method is almost cyclic. The latter can be enforced by e.g. sequentially updating all sensor transmission policies in each iteration (namely, $\mathbf{p}_{1}^{m} \rightarrow \mathbf{p}_{2}^{m} \rightarrow \ldots \rightarrow \mathbf{p}_{I}^{m} \rightarrow \mathbf{p}_{1}^{m+1} \rightarrow \ldots$ ). As for the former, it is worth noting again that the $k$-th term in the summation, namely

$$
G_{k}\left(p_{1, k} \ldots p_{I, k}\right)=\tau_{k} \log \left(1+\left(\sum_{i=1}^{I} \sqrt{p_{i, k}}\right)^{2}\right),
$$


exclusively depends on the vector of transmit powers in the $k$-th epoch. In other words, there are no cross variables. In these circumstances, it suffices to show that for any $k, G_{k}(\cdot)$ is a strictly concave function. This holds true if its Hessian $\nabla^{2} G_{k}$ is negative definite (namely, $\nabla^{2} G_{k} \prec 0$ ) in its domain, that is, if $\mathbf{y}^{T} \nabla^{2} G_{k} \mathbf{y}<0$ for all non-zero vectors $\mathbf{y} \in \mathbb{R}^{I}$.

Let us define functions $f(\cdot)$ and $h(\cdot)$ as

$$
\begin{aligned}
& f(h)=\log (1+h) \\
& h(\mathbf{p})=\left(\sum_{i=1}^{I} \sqrt{p_{i}}\right)^{2}
\end{aligned}
$$

From these definitions, we can write $G(\mathbf{p})=f(h(\mathbf{p}))$ where we have omitted the epoch index $k$ for brevity. Its Hessian can thus be expressed as ${ }^{12}$ :

$$
\nabla^{2} G=\left.\nabla h \frac{\partial^{2} f}{\partial h^{2}}\right|_{h=h(\mathbf{p})} \nabla h^{T}+\left.\frac{\partial f}{\partial h}\right|_{h=h(\mathbf{p})} \nabla^{2} h
$$

with

$$
\begin{gathered}
\nabla h=\left(\sum_{i=1}^{I} \sqrt{p_{i}}\right) \cdot\left[\frac{1}{\sqrt{p_{1}}} \frac{1}{\sqrt{p_{2}}} \cdots \frac{1}{\sqrt{p_{1}}}\right]^{T}, \\
\left.\frac{\partial^{2} f}{\partial h^{2}}\right|_{h=h(\mathbf{p})}=-\frac{1}{\left(1+\left(\sum_{i=1}^{I} \sqrt{p_{i}}\right)^{2}\right)^{2}}, \\
\left.\frac{\partial f}{\partial h}\right|_{h=h(\mathbf{p})}=\frac{1}{1+\left(\sum_{i=1}^{I} \sqrt{p_{i}}\right)^{2}},
\end{gathered}
$$

and

$$
\nabla^{2} h=\frac{1}{2}\left[\begin{array}{ccc}
-\frac{\sum_{i=1}^{I} \sqrt{p_{i}}-\sqrt{p_{1}}}{\sqrt{p_{1}}{ }^{3}} & \ldots & \frac{1}{\sqrt{p_{1}} \sqrt{p_{I}}} \\
\frac{1}{\sqrt{p_{1}} \sqrt{p_{2}}} & \ddots & \frac{1}{\sqrt{p_{2}} \sqrt{p_{I}}} \\
\vdots & \ddots & \vdots \\
\frac{1}{\sqrt{p_{1}} \sqrt{p_{I}}} & \ldots & -\frac{\sum_{i=1}^{I} \sqrt{p_{i}}-\sqrt{p_{I}}}{{\sqrt{p_{I}}}^{3}}
\end{array}\right] .
$$

After some tedious manipulations, it yields

$$
\begin{aligned}
\mathbf{y}^{T} \nabla^{2} G \mathbf{y} & =-\frac{1}{1+h(\mathbf{p})} \cdot[\underbrace{\frac{h(\mathbf{p})}{1+h(\mathbf{p})} \cdot\left(\sum_{i=1}^{I} \frac{y_{i}}{\sqrt{p_{i}}}\right)^{2}}_{L_{1}} \\
& -\underbrace{\frac{1}{2}\left(\sum_{i=1}^{I} \frac{y_{i}}{\sqrt{p_{i}}}\right)^{2}}_{L_{2}} \\
& +\underbrace{\frac{1}{2}\left(\sum_{i=1}^{I} \frac{y_{i}^{2}}{{\sqrt{p_{i}}}^{2}} \cdot \frac{1}{\sqrt{p_{i}}} \sum_{j=1}^{I} \sqrt{p_{j}}\right)}_{L_{3}}]
\end{aligned}
$$

\footnotetext{
${ }^{12}$ The composition of strictly concave functions is not necessarily a strictly concave function [20]. This is why, in the sequel, we derive a specific proof for the function of interest.
}

Interestingly, from Cauchy-Schwartz's inequality [21][Sec. 1.6.2], the following relationship between the $L_{2}$ and $L_{3}$ terms can be established:

$$
\begin{aligned}
L_{3} & =\frac{1}{2}\left(\sum_{i=1}^{I} \frac{y_{i}^{2}}{{\sqrt{p_{i}}}^{2}} \cdot \frac{1}{\sqrt{p_{i}}}\right) \cdot\left(\sum_{j=1}^{I} \sqrt{p_{j}}\right) \\
& \geq \frac{1}{2}\left(\sum_{i=1}^{I} \frac{y_{i}}{\sqrt{p_{i}}} \frac{\sqrt[4]{p_{i}}}{\sqrt[4]{p_{i}}}\right)^{2}=\frac{1}{2}\left(\sum_{i=1}^{I} \frac{y_{i}}{\sqrt{p_{i}}}\right)^{2}=L_{2}
\end{aligned}
$$

where the equality holds if (and only if) $y_{i}=\alpha p_{i}$ for all $i$, that is, if vectors $\mathbf{y}$ and $\mathbf{p}$ are co-linear. Besides, we know from Lemma 3 that all transmit powers $p_{i}$ are strictly positive and, hence, from (33) we have $h(\mathbf{p})>0$. Thus, from (39) it follows that $\nabla^{2} G$ is negative definite iff $L_{1}-L_{2}+L_{3}>0$. Since vector $\mathbf{y}$ is different from zero, this implies $L_{1}, L_{2}>0$, and $L_{3}>0$. If vectors $\mathbf{y}$ and $\mathbf{p}$ are not co-linear then $L_{3}>L_{2}$ and, consequently, $L_{1}-L_{2}+L_{3}>0$. If, on the contrary, vectors $\mathbf{y}$ and $\mathbf{p}$ are co-linear then $L_{2}=L_{3}$ which yields $L_{1}-L_{2}+L_{3}=L_{1}$. Since $y_{i}=\alpha p_{i}$ this means that all $y_{i}$ are either strictly positive or negative. Consequently, $L_{1}>0$ and, again, this yields $\mathbf{y}^{T} \nabla^{2} G \mathbf{y}<0$, which concludes the proof.

\section{Computational Complexity Analysis}

In this section, we analyze the computational complexity of the proposed joint optimization scheme, and compare it with that of the popular interior point methods [22]. More precisely, we focus on the number of operations needed in order to determine the (jointly) optimal transmission policy of one sensor while holding the rest fixed. This renders the comparison independent of the number of iterations of the coordinate descent method described in Section III-A which depends inter-alia on the required precision.

To recall, we construct the jointly optimal transmission policy as the concatenation of a number of feasible BO-like sub-policies. Necessarily, each sub-policy starts and ends in a corner point of its $\mathrm{cEH}$ curve (see Fig. 3). For large $I$, the average number of corner points in each cEH curve reads $P=N / I$. The total number of corner points in the optimal policy is, thus, within the $1 \ldots P$ range. The maximum (worstcase) number of BO-like sub-policies that need to be checked for feasibility (i.e. pseudo-instructions 6 to 15 in Algorithm 1) equals $P+(P-1)+(P-2)+\ldots+1=\frac{P(P-1)}{2}$. The minimum number (best-case) is, clearly, 1 (when the EH sensor behaves as a $\mathrm{BO}$ one), whereas the actual number depends on the specific realization of energy arrivals. In all cases, though, the number of epochs in each sub-policy is upperbounded by $N$. For each of those epochs, the BO-like transmit power is computed according to the iterative procedure given by Algorithm 2 (see Appendix A) which essentially entails solving the third order equation in pseudo-instruction 8. Still, the number of operations that solving it entails, $\Omega$, does not depend on the problem dimensionality (e.g. $N$, or $I$ ). Finally, the total number of times that such third order equation needs to be solved depends on $\chi$, namely, the required accuracy with which the constraint in pseudo-instruction 12 is enforced. When a bi-section scheme is adopted (rather than the grid search actually used in Algorithm 2), the total number of 


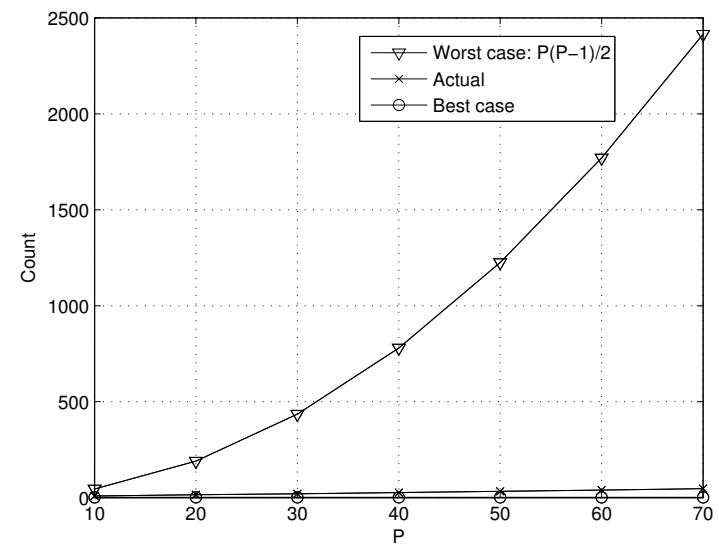

Fig. 5. Number of times that a BO-like sub-policy needs to be checked for feasibility: worst-case, best-case, and actual number averaged over realizations $\left(I=32, N_{c}=1, E_{T}=1 \mathrm{~J}\right)$.

iterations needed scales as $\log \left(\frac{1}{\chi}\right)$ [23]. Bearing all the above in mind, the computational complexity of the proposed scheme is upper-bounded by

$$
\mathcal{O}\left(P^{2} \cdot N \cdot \Omega \cdot \log \left(\frac{1}{\chi}\right)\right)=\mathcal{O}\left(\frac{N^{3}}{I^{2}} \cdot \Omega \cdot \log \left(\frac{1}{\chi}\right)\right)
$$

and lower-bounded by

$$
\mathcal{O}\left(N \cdot \Omega \cdot \log \left(\frac{1}{\chi}\right)\right)
$$

The computational complexity of interior point methods reads [22]

$$
\mathcal{O}\left(N^{3} \cdot \log \left(\frac{1}{\epsilon}\right)\right)
$$

where parameter $\epsilon$ is directly related with the accuracy with which the optimization problem is solved. Clearly, even for the upper bound of (41), the computational complexity savings are on the order of $I^{2}$, for large $N$ (i.e., asymptotically). This is of utmost importance since the number of sensors in such networks is typically high. Furthermore, Figure 5 reveals that the actual number of times that a BO-like policy needs to be checked for feasibility (averaged over realizations) is substantially below the $\frac{P \cdot(P-1)}{2}$ figure of the worst case. For this particular yet representative setting, it was empirically found to be $\mathcal{O}(N P)$. Consequently, the actual savings are much larger than those predicted by the upper bound (41).

\section{Computer Simulation Results}

In this section, we assess the performance of the proposed power allocation algorithm in a scenario where vibrational energy is harvested from the environment. More precisely, sensors are assumed to be deployed along a roadside and the energy they harvest is generated by passing-by vehicles which move at a constant speed. The energy storage device in each sensor comprises (i) a supercapacitor [24]; and (ii) a LithiumIon battery, which is assumed to have infinite capacity. Upon being harvested, the energy is temporarily stored in the supercapacitor. When it is fully charged, the energy is transferred to the battery in a burst which validates the event-based model of the energy harvesting process presented in Section II. For such devices, the amount of energy harvested in each event is constant and it equals the maximum energy that can be stored in the supercapacitor. Except in very dense traffic conditions, the levels of vibrational energy change over time (e.g. when a vehicle passes by), and so does the average number of energy arrivals (events). Consequently, the stochastic process that models energy arrivals is non-stationary. In the sequel, we adopt a Poisson process with time-varying mean given by $\lambda_{E}(t)$. For simplicity, we assume that sensors harvest energy at a constant rate $\lambda_{E}(t)=\lambda_{o}=50 / 32$ when the vehicle is in its vicinity, and $\lambda_{E}(t)=0$ otherwise (i.e. $\lambda_{E}(t)$ is given by a sequence of rectangular pulses).

Hereinafter, we let $E_{T}^{i}=\sum_{k=0}^{N-1} E_{i, k}$ denote the total energy harvested by the $i$-th sensor; whereas $E_{T}=\sum_{i=1}^{I} E_{T}^{i}$ accounts for the total energy in the system. In all plots, we have set $T=320 \mathrm{~s}$. Numerical results are averaged over 1000 realizations of energy arrivals.

\section{A. Two-sensor case}

Here, we assume that two sensors have been deployed at normalized locations $d_{1}=0.05$ and $d_{2} \geq d_{1}$. The normalized inter-sensor distance is denoted by $\Delta d=d_{2}-d_{1}$. Each sensor $i \in\{1,2\}$ is assumed to harvest energy when the passing vehicle is within a road segment centered in $d_{i}$ and total normalized length ${ }^{13} 0.1$. Further, we define $R_{E}=E_{T}^{1} / E_{T}^{2}$ as the ratio between the total energy harvested by the first and second sensors, respectively (i.e. for large $R_{E}$, the first sensor dominates).

In Fig. 6, we depict the throughput attained by the virtual array when using the jointly optimal (JO) transmission policy described in Sections III and IV. We consider scenarios with Gaussian and block Rayleigh-fading channels with channel coherence time $T_{C}=40 \mathrm{~s}$ and identical average SNR. As a benchmark, we consider a system in which the transmission policy for each sensor is separately optimized (SO) as in [1], which is suboptimal for a virtual array. The total energy harvested by each sensor is identical $\left(R_{E}=1\right)$ and the results are shown as a function of normalized inter-sensor distance $\Delta d$. For Gaussian channels and small $E_{T}$, the throughput attained by the JO transmission policy is approximately constant for the whole range of $\Delta d$ values. On the contrary, the performance exhibited by the SO policy degrades when intersensor distance increases: since the first sensor ignores that the second one is idle for most of the time, its harvested energy is mostly wasted before the second sensor starts transmitting. In other words, the JO transmission policy tends to allocate (shift) more energy to the period of time where both sensors are active. The beamforming gain that it entails, results into a higher throughput. For this range of $E_{T}$ values, the larger the inter-sensor distance, the more noticeable this effect becomes. This extent is illustrated for a particular realization in Fig. 7.

On the contrary, for large $E_{T}$ the jointly optimal and suboptimal transmission policies are almost identical ${ }^{14}$, as shown in Fig. 8. This is due to the fact that throughput scales

\footnotetext{
${ }^{13}$ Ultimately, this value depends on the sensitivity of the $\mathrm{EH}$ device

${ }^{14}$ This might not be the case for scenarios where different sensors experience different channel gains (due e.g., to path-loss or fading).
} 


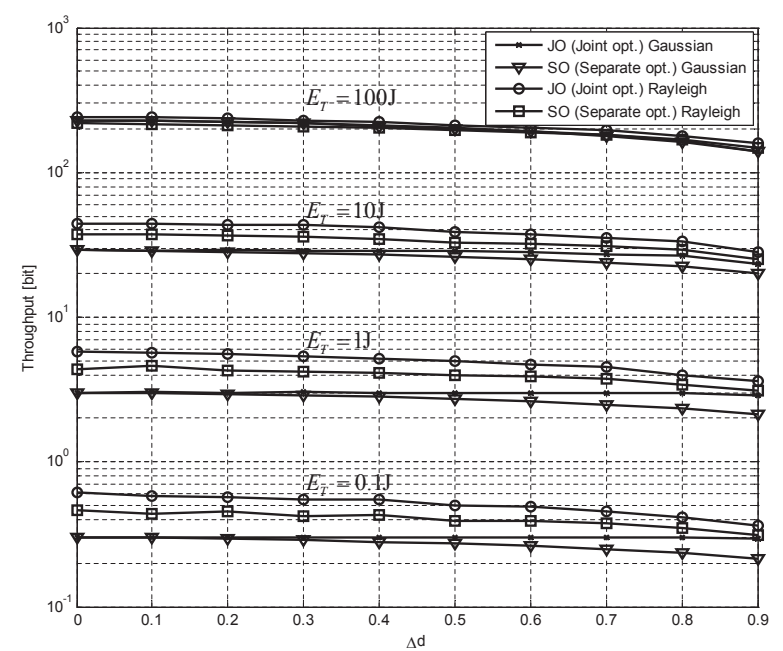

Fig. 6. Throughput vs. normalized inter-sensor distance for several levels of total energy harvested $E_{T}\left(R_{E}=1, I=2\right)$. Gaussian and Rayleigh-fading channels with coherence time $T_{C}=40 \mathrm{~s}$.
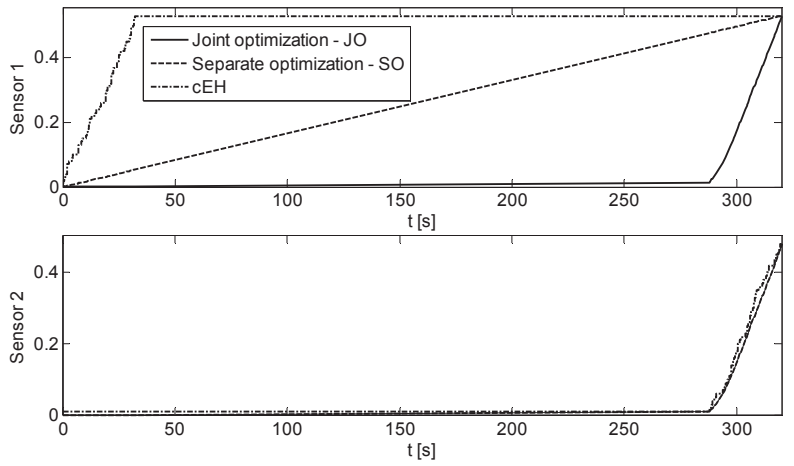

Fig. 7. Joint and separate optimization of transmission policies for one particular realization of energy arrivals $\left(E_{T}=1 J, \Delta d=0.9, I=2\right.$, $\left.R_{E}=1\right)$.

logarithmically in the transmit power (and, thus, on the total harvested energy) and linearly in the transmission time. Hence, for large $E_{T}$ it makes no sense for sensor 1 to wait until sensor 2 starts transmitting: the additional beamforming gain (and instantaneous throughput) cannot compensate the saturation effect of the concave log function and the reduced transmission time. Instead, it is optimal to let sensor 1 transmit for most of the time, as the SO solution does. In other words, joint optimization of transmission policies is particularly useful in the low-SNR regime (i.e. for small $E_{T}$ ). Indeed, for large inter-sensor distances, the throughput achievable by the jointly optimal policy decreases as well, since the contribution of the second sensor vanishes. The larger $E_{T}$, the more noticeable this effect is for smaller values of inter-sensor distance, as Fig. 6 illustrates.

For this simulation setting, the attained throughput in the case of Rayleigh-fading channels turns out to be higher than for Gaussian channels. This stems from the fact that the optimal transmission policy tends to allocate (shift) more power when channel conditions are favorable and cancel transmission otherwise. By doing so, time diversity can be effectively exploited. Unsurprisingly, the gain is higher for the JO policy since, in this case, both time diversity and the gain associated to collaborative transmission can be optimally exploited.
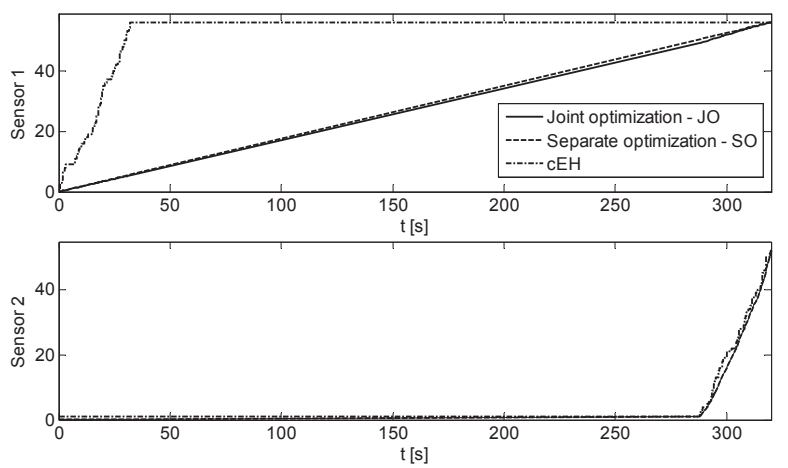

Fig. 8. Joint and separate optimization of transmission policies for one particular realization of energy arrivals $\left(E_{T}=100 J, \Delta d=0.9, I=2\right.$, $\left.R_{E}=1\right)$.

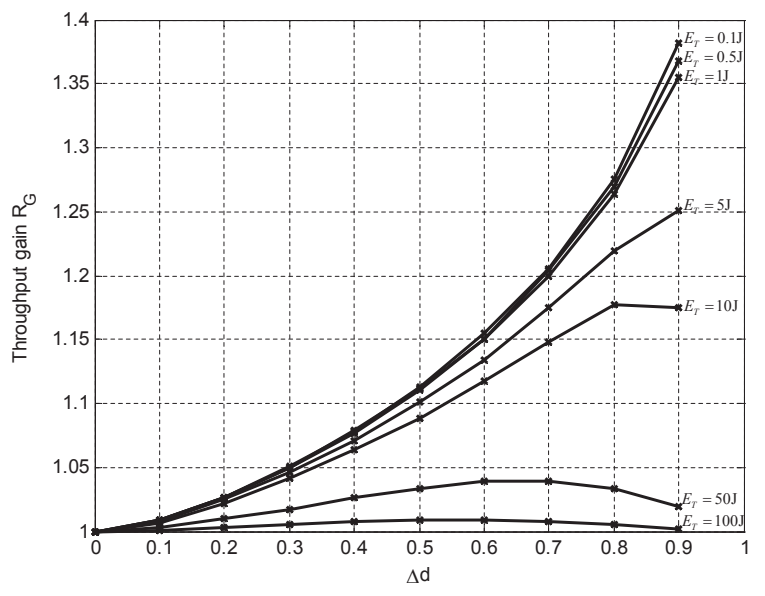

Fig. 9. Throughput gain vs. normalized inter-sensor distance $\left(R_{E}=1\right.$, $I=2)$.

Hereinafter, we focus on results for Gaussian channels only. Fig. 9 provides further insights on the throughput gain stemming from the joint optimization of transmission policies. More precisely, we depict the throughput ratio $R_{G}=\frac{G_{T, \text { opt }}}{G_{T, \text { subopt }}}$ as a function of inter-sensor distance. The throughput gain can be as high as $40 \%$ when the total amount of energy harvested is small $\left(E_{T}=0.1 \mathrm{~J}\right)$ and inter-sensor distance high $(\Delta d=0.9)$. For large $E_{T}$ values and, in particular, in the case of distant sensors, the gain vanishes, as discussed in the previous paragraph.

Next, in Fig. 10, we depict the throughput gain vs. total system energy, for a number of energy ratios $R_{E}=E_{T}^{1} / E_{T}^{2}$ between the first and second sensors. Interestingly enough, the highest gain for most scenarios is attained when the energies harvested by the first and second sensors are identical, that is, for $R_{E}=1$. Yet in a totally different context, this is consistent with [13] where the authors conclude that, in order to maximize the beamforming gain, the received signal levels from the opportunistically selected sensors must be comparable. Conversely, when either the first or second sensor dominate $\left(R_{E} \ll 1\right.$ or $R_{E} \gg 1$, respectively) the gain from the joint optimization becomes marginal $\left(R_{G} \rightarrow 1\right)$ since the signal received from the other sensor is weak. We also observe that, in the case of unbalanced energy levels ${ }^{15}$, the throughput gain is lower when the first sensor dominates. In

\footnotetext{
${ }^{15}$ Such an imbalance might result e.g. from differences in the transduction efficiency of the two sensing devices.
} 


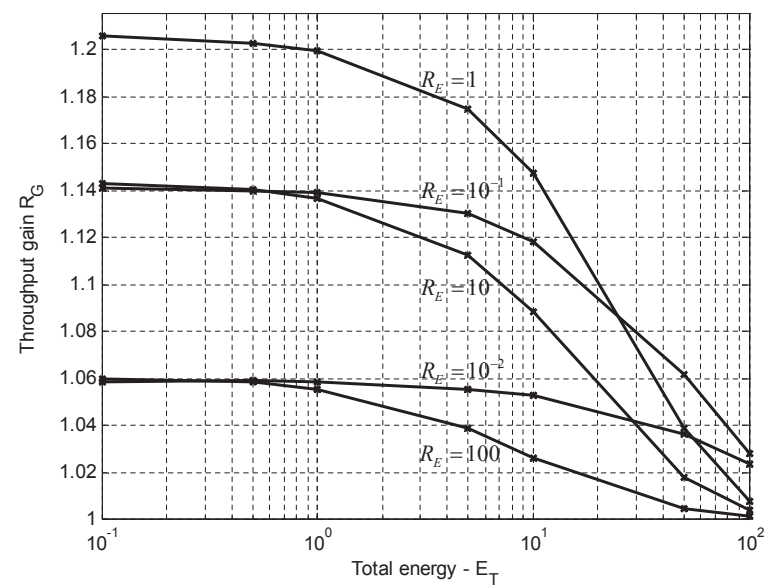

Fig. 10. Throughput gain vs. total energy harvested $(\Delta d=0.7, I=2)$.

other words, when $E_{T}^{1} \gg E_{T}^{2}$ the transmission policy of the second (weak) sensor has very little impact in that of the first (strong) one, which is close to that resulting from a separate optimization over sensors. Finally, and as it was discussed earlier, all throughput gains vanish in the high-SNR regime (i.e. for large $E_{T}$ ).

\section{B. General case}

Hereinafter, we consider a deployment scenario where the $I>2$ sensors in the network are grouped into $N_{c}$ nonoverlapping and homogeneous clusters, each with $I / N_{c}$ sensors. The sensors in the $i$-th cluster are deployed in the vicinity of the normalized location $d_{i}=0.05+i / N_{c}$ for $i=0 \ldots N_{c}-1$ and, again, they harvest energy when the passing vehicle is within a road segment centered in $d_{i}$ and total normalized length equal to 0.1 . It is assumed that all the sensing devices are identical and, thus, we have $E_{T}^{i}=E_{T} / I$ for all $i$. As far as the computation of the transmission policy for each sensor is concerned, we consider four possible strategies, namely,

- Joint Optimization (JO): Here, again, we compute the jointly optimal transmission policy for each sensor as proposed in Section V. Hence, the maximum number of active sensors in the virtual array and, thus, the maximum beamforming gain is $I$. All sensors are allowed to transmit data at any time instant in $[0 \ldots T]$ (of course, as long as some energy has been harvested before). Clearly, this strategy will attain the highest possible throughput. Subsequent ones are suboptimal and will be used as benchmarks.

- Separate Optimization (SO): As in the two-sensor case, here the transmission policy for each sensor is separately optimized (vs. jointly).

- Cluster-by-cluster (CbC): In this strategy, the sensors in the $i$-th cluster are allowed to transmit data until the first sensor in the $i+1$-th cluster becomes active. As a result, (i) the maximum number of simultaneously active sensors is $I / N_{c}$; and, (ii) the maximum transmission time for a specific sensor is, roughly, $T / N_{c}$. That is, the beamforming gain is lower and the transmission time is shorter than in the previous strategies.
TABLE I

OPTIMIZATION MECHANISMS AND PARAMETER SET-UP FOR EACH STRATEGY.

\begin{tabular}{|l|c|c|c|}
\hline Strategy & Optimiz. & Max. Nr. sensors & Max. Tx time \\
\hline Joint Optimization & Joint & $I$ & $T$ \\
Separate Optimization & Separate & $I$ & $T$ \\
Cluster-by-cluster & Joint & $I / N_{c}$ & $T / N_{c}$ \\
All sensors, last cluster & Joint & $I$ & $T / N_{c}$ \\
\hline
\end{tabular}

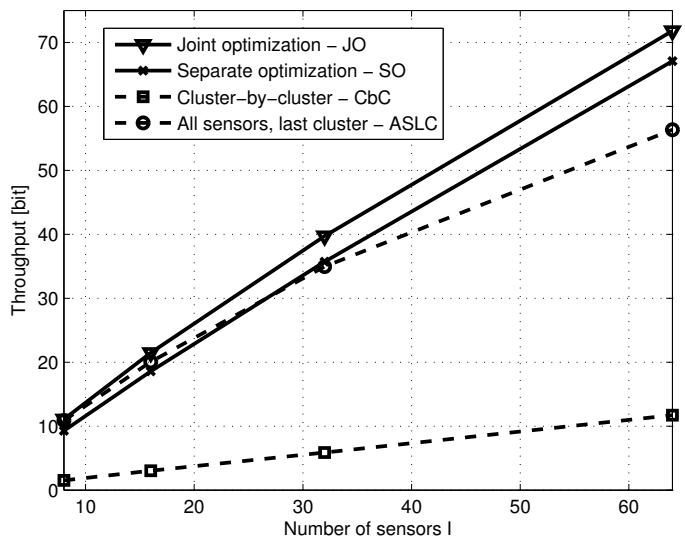

Fig. 11. Throughput vs. number of sensors $\left(N_{c}=8, E_{T}=1 \mathrm{~J}\right)$.

- All sensors, last cluster (ASLC): Here, we assume that the sensors in the first $N_{c}-1$ clusters harvest some energy, they store it in their respective batteries, and postpone data transmissions until the first sensor in the $N_{c}$ cluster harvests some energy (i.e. the time instant in which data transmission for the last cluster can start). As a result, the maximum transmission time for a given sensor is, again, $T / N_{c}$; and the maximum number of active sensors equals $I$.

For the sake of comparison, Table I below summarizes the mechanisms to compute the transmission policy and provides details on the parameter set-up for each strategy. In Fig. 11, we depict the throughput attained by the various strategies in a low to mid-SNR scenario $\left(E_{T}=1 \mathrm{~J}\right)$. Unsurprisingly, throughput is a monotonically increasing function in the number of sensors for all the strategies considered. When the number of sensors increases, so does the beamforming gain and the SNR of the received signal (and, thus, throughput) becomes higher. In other words, by deploying more sensors, we drive the network towards high-SNR regime. Unsurprisingly too, the JO strategy attains the highest throughput. However and as we will see next, the fact that some suboptimal strategies outperform others will ultimately depend on a number of system parameters.

Next, we show some results in terms of the throughput ratio (i.e., loss) between the JO strategy and the suboptimal ones, namely, $L_{G,\{\mathrm{SO}, \mathrm{CbC}, \mathrm{ASLC}\}}=G_{T,\{\mathrm{SO}, \mathrm{CbC}, \mathrm{ASLC}\}} / G_{T, \mathrm{JO}} \leq 1$. In Fig. 12 we depict the corresponding losses as a function of the number of sensors, and for diverse conditions in terms of cluster number and amount of energy harvested. In brief, by moving from (i) the top to the bottom subplot, or (ii) from left to right in each subplot, we drive the system towards the highSNR regime. By moving from the first to the second subplot, we increase the number of clusters as well. Interestingly, 
in the low to mid-SNR regime (top and middle subplots, left), the ASLC strategy outperforms all suboptimal ones and, in particular, separate optimization (SO). Hence, forcing all sensors to simultaneously transmit with those in the last cluster (which leads to an increased beamforming gain) is definitely better than allowing them to transmit at any time in an uncoordinated manner (at the risk of wasting the scarce energy harvested without really acting as a virtual array). As already discussed in the two-sensor case, the performance gap for the JO and SO strategies vanishes in the high-SNR regime. Consequently, $L_{G, \mathrm{SO}} \approx 1$ in the bottom subplot (high $E_{T}$ or in the rightmost part of the top and middle ones. It is also worth noting that, in the low-SNR regime, increasing the number of clusters results into a wider performance gap between the $\mathrm{CbC}$ and ASLC strategies (c.f. upper and middle subplots). Since the number of sensors per cluster is lower and no intercluster coordination takes place (clusters transmit one after the other), this results into a lower beamforming gain for $\mathrm{CbC}$ and, thus, poorer performance (to stress, the maximum transmission time for each sensor is identical in the $\mathrm{CbC}$ and ASLC cases). Finally, we also observe that if we sustainedly drive the sensor network towards higher SNRs (bottom, right), the $\mathrm{CbC}$ strategy finally outperforms ASLC, as the crossing point indicates. Interestingly, this is despite of the fact that the former exhibits a lower beamforming gain $\left(I / N_{c}\right.$ vs. $\left.I\right)$. This, again, is motivated by the fact that throughput is a concave function which increases slowly in the high-SNR regime. In other words, it is more efficient to split the sensors into higher number of clusters and, hence, increase the transmission time linearly $(\mathrm{CbC})$; rather than increasing the beamforming gain and shortening the transmission time (ASLC).

Finally, Fig. 13 provides further insights into the performance of the various strategies as a function of the total energy harvested. The main conclusions are as follows: (i) the JO strategy proposed in this paper is particularly useful for the low-SNR regime; (ii) in the mid-to-high SNR regime, on the contrary, separate optimization results into a marginal loss ( $\mathrm{SO}$ is virtually identical to $\mathrm{JO}$ ); and (iii) the $\mathrm{CbC}$ and ASLC strategies exhibit substantial performance losses in the mid-to-high SNR regime.

\section{CONCLUSIONS}

In this paper, we have proposed a semi-analytical algorithm that allows to compute the jointly optimal transmission policy for a virtual array of energy harvesting sensors in such a way that the throughput for a given deadline is maximized. The optimality of the resulting policy has been rigorously proved. We have also found that the computational complexity of the proposed scheme is upper-bounded by $\mathcal{O}\left(\frac{N^{3}}{I^{2}}\right)$. Consequently, the computational savings with respect to interior point methods are, at least, of an $I^{2}$ factor (yet, in practice, much higher). Performance has been assessed by means of computer simulations in a realistic scenario where vibrational energy is harvested from the environment. Computer simulation results revealed that, in the low-SNR regime, the gain resulting from the joint optimization (vs. separate optimization) of the transmission policies can be as high as $40 \%$ when inter-sensor distance is high. This is achieved by
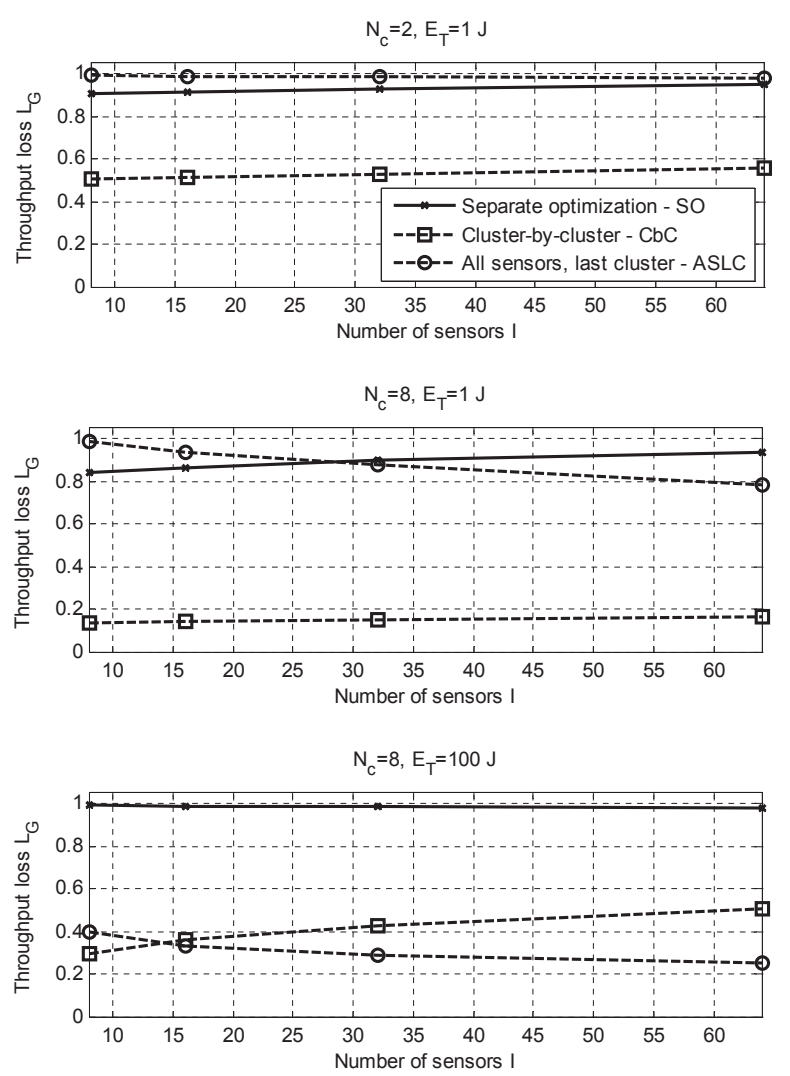

Fig. 12. Throughput ratio (loss) vs. number of sensors. Top: Low-SNR, low number of clusters. Middle: low-SNR, high number of clusters. Bottom: high-SNR, high number of clusters.

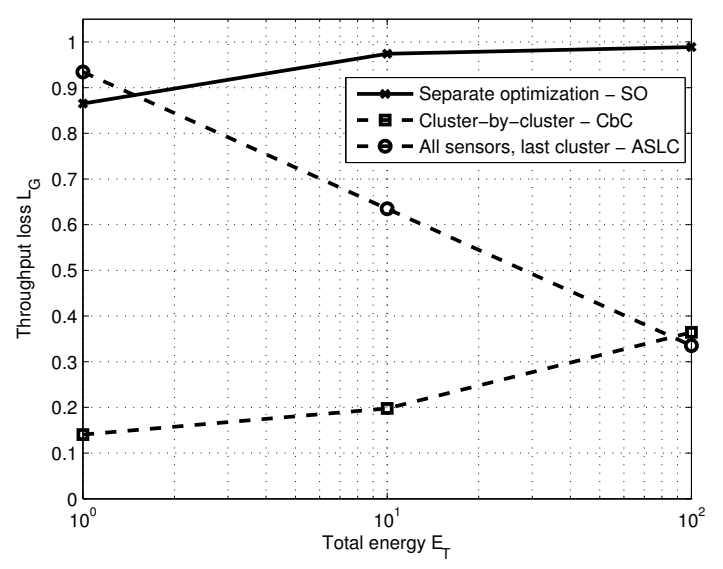

Fig. 13. Throughput ratio (loss) vs. total energy $E_{T}\left(N_{c}=8, I=16\right)$.

favoring simultaneous sensor transmissions which result into a higher beamforming gain (and, thus, throughput). On the contrary, in the high-SNR regime the jointly and separately optimal transmission policies are almost identical and, hence, such gain vanishes. This follows from the fact that throughput scales logarithmically in the transmit power and linearly in the transmission time. We have also concluded that, for most scenarios, the throughput is higher when each sensor in the virtual array harvests the same amount of total energy. As for the other suboptimal transmissions strategies (cluster-bycluster, all sensors-last cluster), we have learnt that, in the low-to-mid SNR regime the all sensors-last cluster policy outperforms all the rest and, in particular, separate optimization. 
Besides, the performance gap between between the clusterby-cluster and all sensors-last cluster strategies becomes wider when the number of cluster increases. In the high-SNR regime, on the contrary, the cluster-by-cluster strategy outperforms the all sensors-last cluster one.

\section{APPENDIX A}

\section{COMPUTATION OF THE JOINTLY OPTIMAL TRANSMISSION POLICY FOR A BATTERY OPERATED SENSOR}

Consider a scenario where one energy harvesting $(\mathrm{EH})$ plus one battery operated (BO) sensor cooperate to transmit a common message to a distant base station. In [14], we proved that upon finding the optimal transmission policy for the EH sensor, $\left\{\breve{p}_{k}^{H}\right\}_{k=1}^{N}$, the jointly optimal transmission policy for the BO sensor, $\left\{\breve{p}_{k}^{B}\right\}_{k=1}^{N}$, can be computed with the iterative procedure given by Algorithm 2. Since $\breve{p}_{k}^{H}$ is

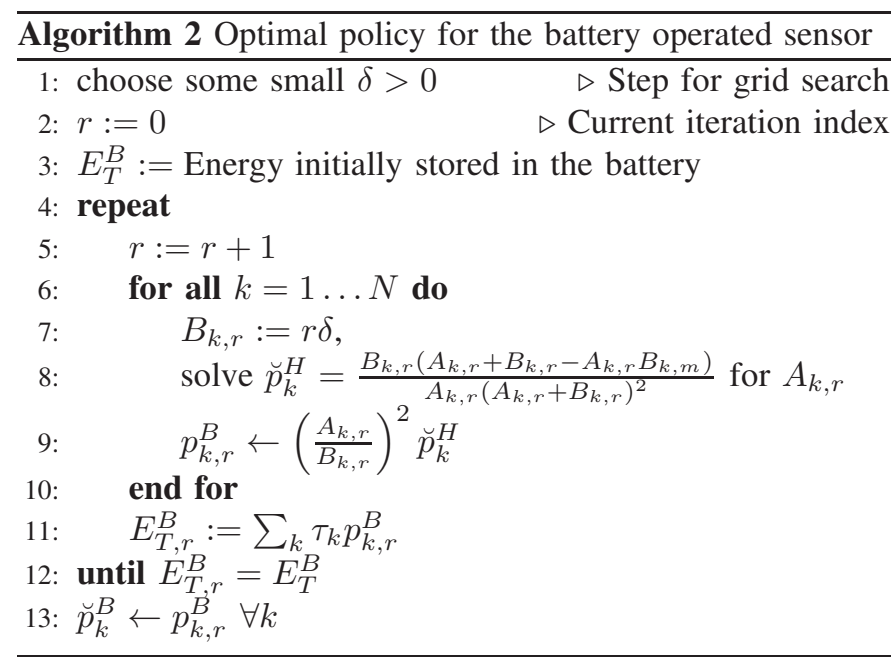

already known, for each value of $B_{k, r}$ to be tested (which is computed in pseudo-instruction 7), the associated $A_{k, r}$ can be found by solving the corresponding third order equation in pseudo-instruction 8 (a single real-valued and positive root exists). From $\breve{p}_{k}^{H}, A_{k, r}$, and $B_{k, r}$, an estimate of the optimal transmission policy for the battery operated sensor for the current iteration, namely, $\left\{p_{k, r}^{B}\right\}_{k=1}^{N}$, follows in pseudoinstruction 9. If the total energy consumed until time instant $T$ by the battery operated sensor, computed in pseudo-instruction 11 , equals the energy (initially) stored in it, $E_{T}^{B}$, the iterative algorithm stops. The stopping condition not only enforces Lemma 2 but, as discussed in [14], it also guarantees that the whole transmission policy for the battery operated sensor $\left\{\breve{p}_{k}^{B}\right\}_{k=1}^{N}$ is feasible. Clearly, the choice of $\delta$ leads to a number of trade-offs in terms of accuracy vs. number of iterations needed.

\section{APPENDIX B \\ PROOF OF LEMMA 4}

Consider two candidate transmission policies, $\mathbf{p}_{1}$ and $\dot{\mathbf{p}}_{1}$, the first $L$ or $R$ (respectively) elements of which, namely, $\mathbf{p}_{1,1: L}$ and $\dot{\mathbf{p}}_{1,1: R}$ with $R<L$, have been computed as BOlike solutions (see Fig. 14). From the discussions in Steps 1

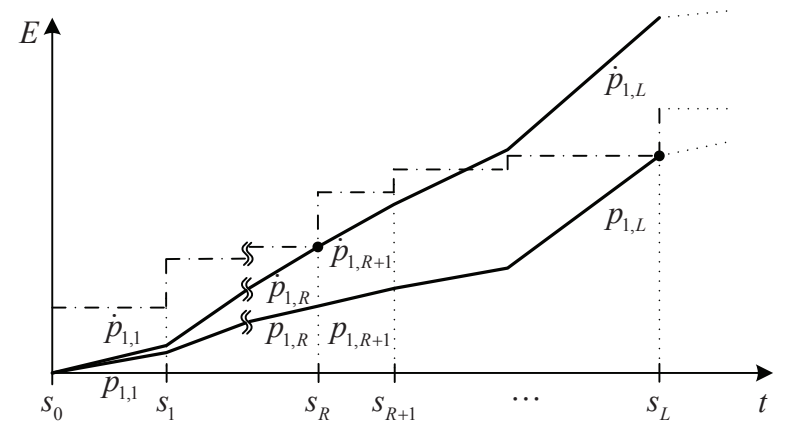

Fig. 14. Lemma 4: A sub-policy consuming all the harvested energy in $s_{R}$ is not feasible and, thus, is not part of the overall optimal policy.

and 2 above, we know that $\mathbf{p}_{\mathbf{1}}$ verifies

$$
\lambda_{n}=0, n=1, \ldots, L-1 ; \lambda_{L}>0
$$

and, as long as $L<N$,

$$
\lambda_{n} \geq 0, n=L+1, \ldots, N
$$

where (44) follows from the K.K.T. conditions. From this, the corresponding sums of Lagrangian multipliers $A_{k}$ verify in turn

$$
A_{1}=A_{2}=\ldots=A_{L}=\sum_{n=L}^{N} \lambda_{n} .
$$

Likewise, for $\dot{\mathbf{p}}_{1}$ we have

$$
\begin{aligned}
& \dot{\lambda}_{n}=0, n=1, \ldots, R-1 ; \dot{\lambda}_{R}>0 \\
& \dot{\lambda}_{n} \geq 0, n=R+1, \ldots, N
\end{aligned}
$$

and, hence,

$$
\dot{A}_{1}=\dot{A}_{2}=\ldots=\dot{A}_{R}=\sum_{n=R}^{N} \dot{\lambda}_{n} .
$$

Since in $s_{R}$ the EC curve associated to $\dot{\mathbf{p}}_{1}$ lies above that of $\mathbf{p}_{1}$ (see Fig. 14), there necessarily exists at least one epoch $k_{o} \in\{1 \ldots R\}$ such that $\dot{p}_{1, k_{o}}>p_{1, k_{o}}$. Besides, a necessary condition for either candidate sub-policy to qualify as optimal, is that the corresponding derivative of the Lagrangian given by (25) must vanish for all $k \in\{1 \ldots R\}$, namely, $\frac{\partial \mathcal{L}_{1}}{\partial \breve{p}_{1, k}}=0$. In particular, for $k=k_{o}$ the fact that $\dot{p}_{1, k_{o}}>p_{1, k_{o}}$ implies that $\dot{A}_{k_{o}}<A_{k_{o}}$ (and vice-versa: if $\dot{p}_{1, k_{o}}<p_{1, k_{o}}$ then $\dot{A}_{k_{o}}>A_{k_{o}}$ ). Moreover, from (45) and (47), we conclude that $\dot{A}_{k}<A_{k}$ for all $k \in\{1 \ldots R\}$ which, in turn, implies $\dot{p}_{1, k}>p_{1, k}$ for those epochs. In other words, the EC curve associated to the candidate sub-policy $\dot{\mathbf{p}}_{1,1: R}$ lies strictly above that of $\mathbf{p}_{1,1: R}$ for all those epochs, as Fig. 14 illustrates. This holds true in particular for $k=R$, namely, $\dot{p}_{1, R}>p_{1, R}$. For the next epoch, we have that either $\dot{p}_{1, R+1}<p_{1, R+1}$ or $\dot{p}_{1, R+1} \geq p_{1, R+1}$. The former would hold if (and only if)

$$
\begin{gathered}
\dot{A}_{R}<A_{R}=A_{L} \\
\dot{A}_{R+1}>A_{R+1}=A_{L}
\end{gathered}
$$

which renders $\dot{\lambda}_{R}=\dot{A}_{R}-\dot{A}_{R+1}<0$. Since this contradicts one K.K.T. condition then, necessarily, $\dot{p}_{1, R+1} \geq p_{1, R+1}$. By applying the same reasoning to the subsequent consecutive epoch pairs, we conclude that $\dot{p}_{1, k} \geq p_{1, k}$ for all $k \in\{R+$ 
$1, L\}$. Hence, the EC curve associated to the candidate subpolicy $\dot{\mathbf{p}}_{1,1: L}$ lies strictly above that of $\mathbf{p}_{1,1: L}$ not only for the first $R$ epochs but for all epochs $k \in\{1 \ldots L\}$. Since, to recall, at $s_{L}$ the EC associated to the candidate sub-policy $\dot{\mathbf{p}}_{1,1: L}$ hits the cEH curve, this means that the candidate subpolicy $\dot{\mathbf{p}}_{1,1: L}$ is necessarily not feasible and, thus, cannot be part of the overall optimal policy (see Fig. 14). This concludes the proof.

\section{REFERENCES}

[1] J. Yang and S. Ulukus, "Optimal packet scheduling in an energy harvesting communication system," IEEE Trans. Commun., vol. 60, no. 1 , pp. $220-230$, january 2012 .

[2] K. Tutuncuoglu and A. Yener, "Optimum transmission policies for battery limited energy harvesting nodes," IEEE Trans. Wireless Commun., vol. 11, no. 3, pp. $1180-1189$, march 2012.

[3] O. Ozel, K. Tutuncuoglu, J. Yang, S. Ulukus, and A. Yener, "Transmission with energy harvesting nodes in fading wireless channels: Optimal policies," IEEE J. Sel. Areas Commun., vol. 29, no. 8, pp. 1732 -1743, september 2011.

[4] J. Yang and S. Ulukus, "Optimal packet scheduling in a multiple access channel with energy harvesting transmitters," Journal of Communications and Networks, vol. 14, no. 2, pp. 140 -150, april 2012.

[5] K. Tutuncuoglu and A. Yener, "Sum-rate optimal power policies for energy harvesting transmitters in an interference channel," Journal of Communications and Networks, vol. 14, no. 2, pp. 151 -161, april 2012.

[6] C. Huang, R. Zhang, and S. Cui, "Throughput maximization for the Gaussian relay channel with energy harvesting constraints," IEEE J. Sel. Areas Commun., vol. 31, no. 8, pp. 1-11, 2013.

[7] O. Orhan and E. Erkip, "Energy harvesting two-hop networks: Optimal policies for the multi-energy arrival case," in 35th IEEE Sarnoff Symposium (SARNOFF), 2012, pp. 1-6.

[8] J. Yang, O. Ozel, and S. Ulukus, "Broadcasting with an energy harvesting rechargeable transmitter," IEEE Trans. Wireless Commun., vol. 11, no. 2, pp. $571-583$, february 2012.

[9] O. Ozel, J. Yang, and S. Ulukus, "Optimal broadcast scheduling for an energy harvesting rechargeable transmitter with a finite capacity battery," IEEE Trans. Wireless Commun., vol. 11, no. 6, pp. 2193 -2203, june 2012.

[10] B. Devillers and D. Gunduz, "A general framework for the optimization of energy harvesting communication systems with battery imperfections," Journal of Communications and Networks, vol. 14, no. 2, pp. $130-139$, april 2012.

[11] K. Tutuncuoglu and A. Yener, "Communicating using an energy harvesting transmitter: Optimum policies under energy storage losses," 2012. [Online]. Available: "http://arxiv.org/pdf/1208.6273v1.pdf"

[12] R. Mudumbai, J. Hespanha, U. Madhow, and G. Barriac, "Distributed transmit beamforming using feedback control," IEEE Trans. Inf. Theory, vol. 56, no. 1, pp. $411-426$, jan. 2010.

[13] M.-O. Pun, D. Brown, and H. Vincent Poor, "Opportunistic collaborative beamforming with one-bit feedback," SPAWC Workshop 2008., pp. 246 -250 , jul. 2008.

[14] L. Berbakov, C. Anton-Haro, and J. Matamoros, "Optimal transmission policy for cooperative transmission with energy harvesting and battery operated sensor nodes," Signal Processing, vol. 93, no. 11, pp. $3159-$ 3170, 2013. [Online]. Available: http://www.sciencedirect.com/science/ article/pii/S0165168413001515

[15] N. Liu and S. Ulukus, "Capacity region and optimum power control strategies for fading Gaussian multiple access channels with common data," IEEE Trans. Commun., vol. 54, no. 10, pp. 1815-1826, 2006.

[16] Z.-Q. Luo and P. Tseng, "On the convergence of the coordinate descent method for convex differentiable minimization," Laboratory for Information and Decision Systems, Massachusetts Institute of Technology, dec. 1989

[17] L. Berbakov, J. Matamoros, and C. Anton-Haro, "Optimal transmission policy for distributed beamforming with energy harvesting and battery operated sensor nodes," in ISWCS 2012, aug. 2012, pp. $441-445$.

[18] D. P. Bertsekas and J. N. Tsitsklis, Parallel and Distributed Computation: Numerical Methods. Athena Scientific, 1989.

[19] O. Kaya and S. Ulukus, "Achieving the capacity region boundary of fading CDMA channels via generalized iterative waterfilling," IEEE Trans. Wireless Commun., vol. 5, no. 11, pp. 3215-3223, 2006.
[20] S. Boyd and L. Vandenberghe, Convex Optimization. Cambridge University Press, 2004

[21] A. D. Polyanin and A. V. Manzhirov, Handbook of Mathematics for Engineers and Scientists. Chapman \& Hall/CRC, 2007.

[22] Y. Ye, Interior Point Algorithms: Theory and Analysis. John Wiley \& Sons, Inc, 1997.

[23] R. L. Burden and J. D. Faires, Numerical Analysis. Brooks/Cole, 2011.

[24] S. Saggini, F. Ongaro, C. Galperti, and P. Mattavelli, "Supercapacitorbased hybrid storage systems for energy harvesting in wireless sensor networks," in APEC 2010, feb. 2010, pp. $2281-2287$. 